\title{
An abnormally positioned and morphologically variant sigmoid colon: case report
}

\author{
Dawit Habte Woldeyes, Abebe Ayalew Bekel, Shibabaw Tedila Tiruneh, Yibeltal Wubale Adamu \\ Department of Human Anatomy, College of Medicine and Health Sciences, Babir Dar University, Babir Dar, Ethiopia
}

\begin{abstract}
The sigmoid colon is about $40 \mathrm{~cm}$ in length and begins below the pelvic inlet and ends at the rectosigmoid junction. It normally lies in the lesser pelvis. Anatomical variations of the sigmoid colon were described by various authors. The length and form of sigmoid colon are the most variable of all colonic segments. In this case, the sigmoid colon was found covering the left half of the transverse colon, hiding the spleen and in contact with the left lobe of the liver. In addition, it had no visible taeniae coli and sacculations. The sigmoid colon was $66 \mathrm{~cm}$ in length, $7 \mathrm{~cm}$ in lumen width, and in suprapelvic position. Awareness of the possible variation of colon especially of the sigmoid is necessary for adequate clinical, surgical and radiological management.
\end{abstract}

Keywords: length; morphology; position; sigmoid colon; variation

Anatomy 2017;11(3):153-156 (2017 Turkish Society of Anatomy and Clinical Anatomy (TSACA)

\section{Introduction}

The sigmoid colon, also named the pelvic colon, is the continuation of the descending colon that begins at the left pelvic brim and ends at the rectosigmoid junction in front of the third sacral vertebra. It normally lies in the lesser pelvis and is about $40 \mathrm{~cm}$ in length. The sigmoid loop may arise out of the pelvic in the abdominal cavity and lie in contact with loop of ileum. ${ }^{[1-3]}$

The sigmoid colon forms a mobile loop which lies in the lesser pelvis, but its length and form are the most variable of all colonic segments. It may remain folded and principally in contact with, or adherent to, the peritoneum overlying the iliacus musscle, or it may cross the pelvic cavity between the rectum and bladder in males, or the rectum and uterus in females, and it may even reach the right pelvic wall. ${ }^{[1,3,4]}$

The position and shape of the sigmoid colon may vary according to the length of the colon, the length and mobility of its mesocolon, the degree of distension, the condition of the rectum, bladder and uterus. ${ }^{[2,4]}$ Sigmoid colon morphology also depends on age, gender, geographical variation, diet, or defecation habits. ${ }^{[5]}$
The length and diameter of the sigmoid colon vary in different races. Longer sigmoid colon and mesocolon were reported in African population, which was attributed as one of the causes of sigmoid volvulus. ${ }^{[3,4,6]}$ The length of the sigmoid colon increases with age, and males have longer sigmoid colon than females. ${ }^{[7]}$ Studies have shown that variation in the shape of mesocolon can alter the morphology of sigmoid colon. ${ }^{[3]}$

Variations in the position and length of the sigmoid colon may produce difficulties in radiological diagnosis and instrumentation. They may also form volvulus or result in some other functional disturbances in the neighboring structures. ${ }^{[2]}$

The sigmoid colon is a site for many acquired pathological conditions and congenital anomalies like carcinomas, volvulus, colonic varices, diverticulosis, ulcerative colitis, epiploic appendices, diverticulitis, congenital megacolon, atresia and duplication. ${ }^{[1,5]}$ Knowledge of the variation in the morphology and position of the sigmoid colon is of value to clinicians, pathologists, surgeons and radiologists for interventional procedures, preventing misdiagnosis and treatment of an abnormally positioned sigmoid colon. 
Sigmoid colon morphology varies with geography and race; therefore, the main objective of the present paper was to report a morphologically variant sigmoid colon that was not reported earlier in Ethiopia that was observed in a male cadaver dissected in routine gross anatomy course. Possible clinical and functional implications of the anomalous positions of the sigmoid colon were discussed.

\section{Case Report}

During routine dissection of a formaldehyde fixed cadaver for undergraduate medical students in the Department of Human Anatomy, College of Medicine and Health Sciences, Bahir Dar University, Bahir Dar, Ethiopia, an unusually placed and morphologically different type of aigmoid colon was observed in a 45-year-old male Ethiopian cadaver. The hospital of the College of Medicine and Health Sciences reported that the cause of death was not related to the detected anomaly. Past medical history indicated no pathological processes related to the digestive system, no traumatic injuries of the region, and no previous surgical interventions.

The dissection was performed, as regularly, according to the Cunningham's Manual of Practical Anatomy, Volume $\mathrm{II}^{[8]}$ for 1 st year preclinical medical students in the College of Medical Sciences, Bahir Dar University. With the body placed in supine position, the anterior abdominal wall was dissected and after the reflection of the anterolateral abdominal wall and removal of the peritoneum, abdominal organs were observed for their loca- tions in the abdominal cavity and the following new features were noted (Figure 1). The sigmoid colon was found covering the left half of the transverse colon anteriorly, it also hided the spleen and was in contact with the left lobe of the liver. Normally, the sigmoid colon is expected to be located in the pelvis below the pelvic brim. In addition, the sigmoid colon had a smooth external wall - no visible taeniae coli - no sacculations as evident in other parts of the colon. We also observed that it also had a thin wall and wide lumen measuring $7 \mathrm{~cm}$, especially in the middle part of it (Figure 2). The length of the sigmoid colon was $66 \mathrm{~cm}$; in classical textbooks, its length is usually described as $40 \mathrm{~cm}$.

\section{Discussion}

Anatomy textbooks describe the sigmoid colon as a visceral organ with constant morphology, but with variations in its length. Various authors reported the variant morphology of the sigmoid colon. Appropriate knowledge of these variations other than those quoted in classical textbooks is important from both medical and surgical aspects.

Madiba and Haffajee ${ }^{[9]}$ classified the sigmoid colon into three main types: classical, long- narrow and long- broad type. The classical type of sigmoid colon is the one that lies close to the pelvic brim with the normal anatomical textbook description. In the long-narrow type, sigmoid colon is long with the root of mesocolon being narrow; in the long-broad type, though the sigmoid colon is long, the mesocolon is broad.

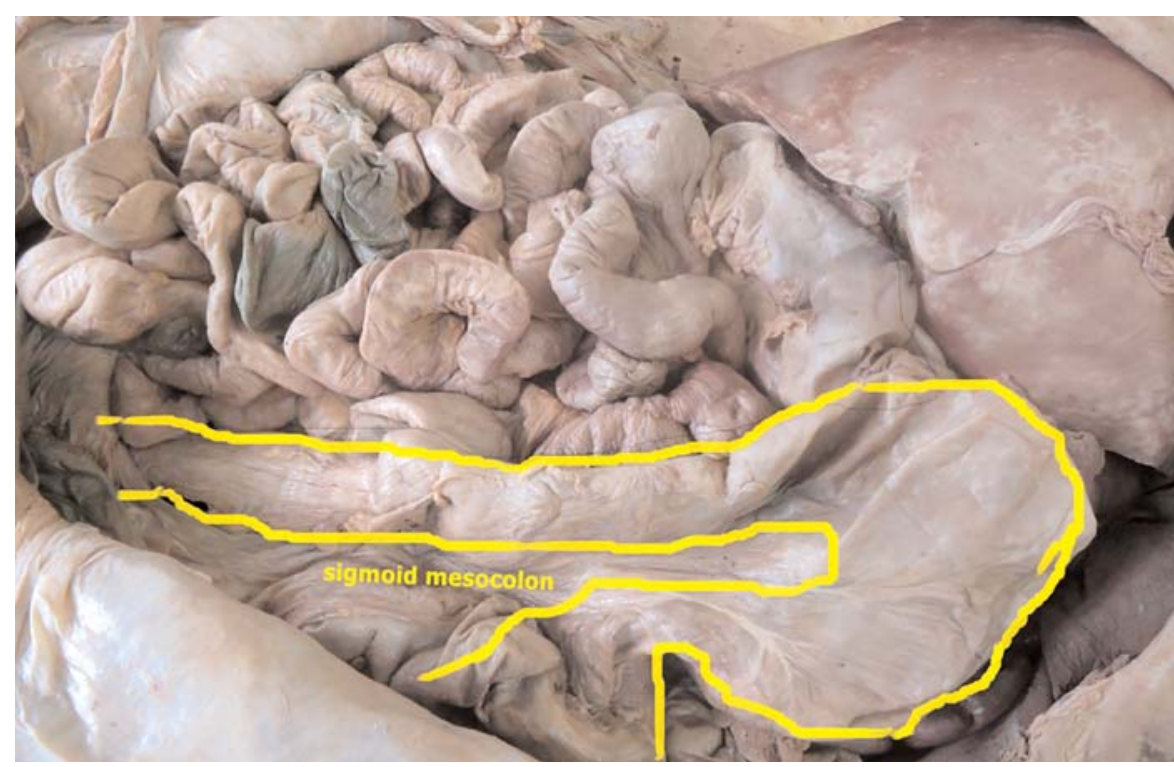

Figure 1. Sigmoid colon taken in suprapelvic position, in contact with the transverse colon. [Color figure can be viewed in the online issue, which is available at www.anatomy. org.tr] 
In our case, the sigmoid colon was found covering the left half of the transverse colon, hiding the spleen and in contact with the left lobe of the liver; in addition, it had a wide lumen, and no visible teniae coli and haustra. This report matches with the long-narrow type description of Madiba and Haffajee..$^{[9]}$ Madiba and Haffajee also classified the sigmoid colon in reference to the pelvic brim as pelvic and suprapelvic positions $;{ }^{[9]}$ our case falls in to the category of suprapelvic type.

Nayak et al..$^{[2]}$ reported a case of sigmoid colon where it occupied the median position in the lesser pelvis, completely filling the rectovesical pouch and covering the superior aspect of the urinary bladder. This report is similar to our report in that it is a misplaced sigmoid colon, but different in other morphologies: suprapelvic in this case and infrapelvic in Nayak's case.

Textbooks and some authors in the literature refer to the average length of the sigmoid colon as $40 \mathrm{~cm} \cdot{ }^{[2,4]}$ Cunningham's Manual of Practical Anatomy describes the length of the sigmoid colon as $16-80 \mathrm{~cm} \cdot{ }^{[8]}$ The length was significantly higher in males when compared with females and considerably more in Africans. ${ }^{[3,6,10]}$ In this case, we found the sigmoid colon as $66 \mathrm{~cm}$ in length and $7 \mathrm{~cm}$ in diameter. A study performed in Nigeria in 50 fresh cadavers showed that the mean length of the sigmoid colon was $50.1 \pm 1.6 \mathrm{~cm}$ (ranging $34.5-67.8 \mathrm{~cm}$ ). ${ }^{[7]}$ Bhatnagar et al. ${ }^{[11]}$ measured the length as $46.6 \mathrm{~cm}$, Madiba et al ${ }^{[6]}$ as $57 \mathrm{~cm}$. A study from Turkey by Atamanalp et al. ${ }^{[5]}$ measured the sgmoid colon length as $43.7 \mathrm{~cm}$. No significant correlation was reported between the length of sigmoid colon and its various types in the literature.
The morphology sigmoid mesocolon in our case was dolichocholic, similar to the study of Atamanalp et al., ${ }^{[5]}$ Akinkuotu et al., ${ }^{[7]}$ and Bhatnagar et al. ${ }^{[11]}$ (Figure 1). In our case, the sigmoid colon had a smooth external wall with no taeniae coli and haustra; but we did not find any literature with similar morphology for further comparison.

The primary ontogenic factors responsible for positional variations of the colon are the differential development of abdominal organs with their peritoneal coverings and a mechanical factor. ${ }^{[12]}$ During retraction of the intestinal loop (large intestine) into the abdominal cavity, the dorsal mesenteries of the ascending colon and descending colon shorten and fold, bringing these organs into contact with the dorsal body wall where they adhere and become secondarily retroperitoneal. The transverse colon and the sigmoid do not become fixed to the body wall, but remain as an intraperitoneal organ suspended by mesentery. ${ }^{[13]}$ However, the sigmoid mesocolon is very long, so that it is a mobile part of the gastrointestinal tract contributing to its positional changes.

\section{Conclusion}

Knowledge of variations of the position and morphology of sigmoid colon contribute to the existing information of the variations in the colon and success of surgical, invasive and radiological procedures of this area. Variations in this region should not be overlooked, because these anatomical findings can help in understanding the disease process related to the colon. Since sigmoid morphology varies with geography and race, further studies on the sgigmoid colon should be performed in Ethiopia.

Figure 2. Sigmoid colon taken with its relationship with the neighbouring structures, and the width of the lumen. $\mathrm{d}$ : diameter; SI: small intestine; Tr: transverse. [Color figure can be viewed in the online issue, which is available at www.anatomy.org.tr]

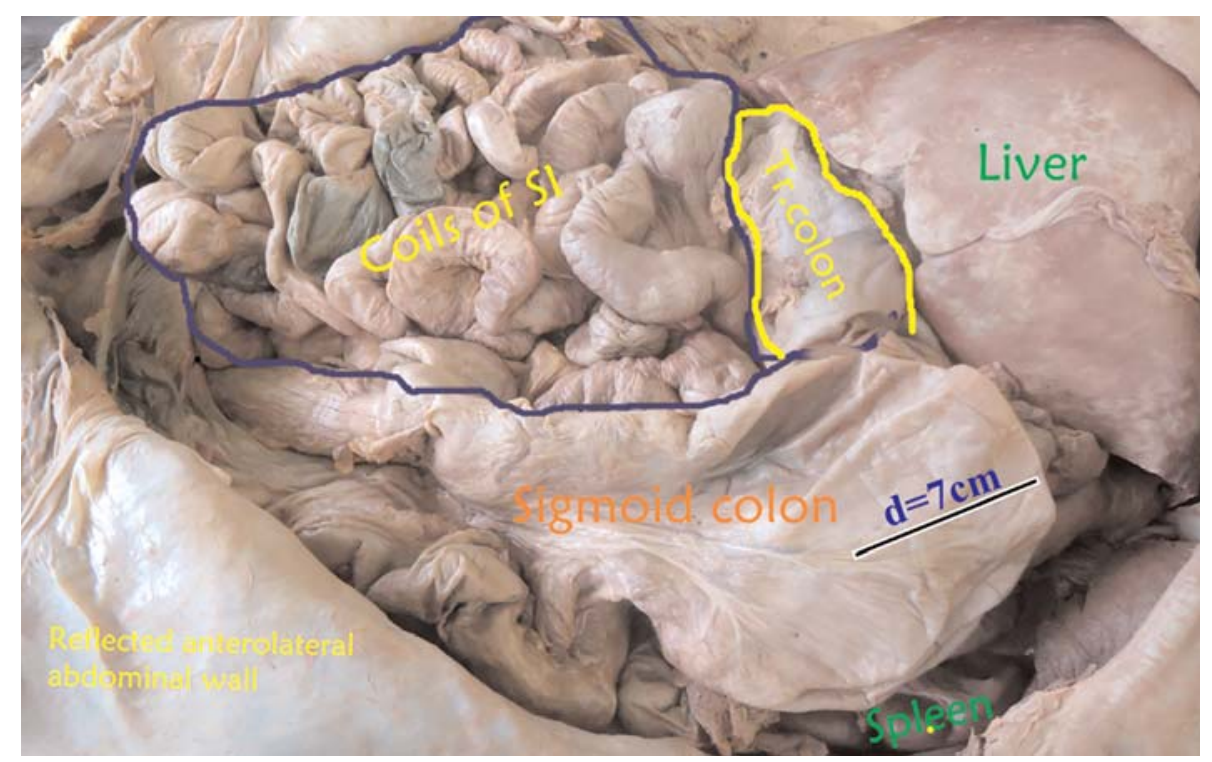




\section{References}

1. Syamala G, Prasad K. Anatomical study of sigmoid colon. IOSR Journal of Dental and Medical Sciences 2016;15:26-30.

2. Nayak SB, Pamidi N, Shetty SD, Sirasanagandla R, Ravindra SS, Guru A, Kumar N. Displaced sigmoid and descending colons: a case report. OA Case Reports 2013;2:166.

3. Michael SA, Rabi S. Morphology of sigmoid colon in South Indian population: a cadaveric study. J Clin Diagn Res 2015;9:AC047.

4. Standring S. Gray's Anatomy: the anatomical basis of clinical practice. 40th ed. London (UK): Churchill Livingstone Elsevier; 2008. p. $1147-8$.

5. Atamanalp SS, Öztürk G, Aydınlı B, Ören D. The relationship of the anatomical dimensions of the sigmoid colon with sigmoid volvulus. Turk J Med Sci 2011;41:377-82.

6. Madiba TE, Haffajee MR, Sikhosana MH. Radiological anatomy of the sigmoid colon. Surg Radiol Anat 2008;30:409-15.
7. Akinkuotu A, Samuel JC, Msiska N, Mvula C, Charles AG. The role of the anatomy of the sigmoid colon in developing sigmoid volvulus: a case-control study. Clin Anat 2011;24:634-7.

8. Romanes GJ. Cunningham's manual of practical anatomy: the abdomen. New York (NY): Oxford Medical Publications; 2010. p. 175-86.

9. Madiba TE, Haffajee MR. Sigmoid colon morphology in the population groups of Durban, South Africa, with special reference to sigmoid volvulus. Clin Anat 2011;24:441-53.

10. Sadahiro S, Ohmura T, Yamada Y, Saito T, Taki Y. Analysis of length and surface area of each segment of the large intestine according to age, sex and physique. Surg Radiol Anat 1992;14:251-7.

11. Bhatnagar BN, Sharma CL, Gupta SN, Mathur MM, Reddy DC. Study on the anatomical dimensions of the human sigmoid colon. Clin Anat 2004; 17: 236-43.

12. Hadar H, Gadoth N. Positional relations of colon and kidney determined by perirenal fat. AJR Am J Roentgenol 1984;143:773-6.

13. Schoenwolf GC, Bleyl SB, Brauer PR, Francis-West PH. Larsen's human embryology. 4th ed. Philadelphia (PA): Churchill Livingstone Elsevier; 2009. p. 456.

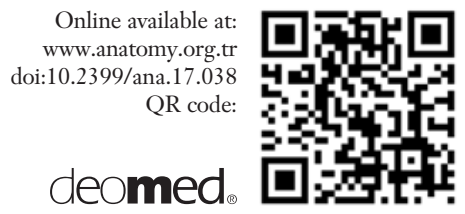

Correspondence to: Dawit Habte Woldeyes, MSC Department of Human Anatomy, College of Medicine and Health Sciences, Bahir Dar University, Bahir Dar, Ethiopia Phone: +251938221383

e-mail: dwthabte@gmail.com

Conflict of interest statement: No conflicts declared.

This is an open access article distributed under the terms of the Creative Commons Attribution-NonCommercial-NoDerivs 3.0 Unported (CC BY-NCND3.0) Licence (http://creativecommons.org/licenses/by-nc-nd/3.0/) which permits unrestricted noncommercial use, distribution, and reproduction in any medium, provided the original work is properly cited. Please cite this article as: Woldeyes DH, Bekel AA, Tiruneh ST, Adamu YW. An abnormally positioned and morphologically variant sigmoid colon: case report. Anatomy 2017;11(3):153-156. 